\title{
Resummation for Top Quark Pair Production at the LHC
}

Michał Czakon, ${ }^{b}$ Andrea Ferroglia, ${ }^{c}$ David Heymes, ${ }^{d}$ Alexander Mitov, ${ }^{d}$

Ben D. Pecjak, ${ }^{e}$ Darren J. Scott', ${ }^{*}$ Xing Wang $^{a}$ and Li Lin Yang ${ }^{a, f, g}$

${ }^{a}$ School of Physics and State Key Laboratory of Nuclear Physics and Technology

Peking University, Beijing 100871, China

${ }^{b}$ Institut für Theoretische Teilchenphysik und Kosmologie, RWTH Aachen University

D-52056 Aachen, Germany

${ }^{c}$ New York City College of Technology, 300 Jay Street

Brooklyn, NY 11201, USA

${ }^{d}$ Cavendish Laboratory, University of Cambridge

CB3 OHE Cambridge, UK

${ }^{e}$ Institute for Particle Physics Phenomenology, University of Durham

DH1 3LE Durham, UK

${ }^{f}$ Collaborative Innovation Center of Quantum Matter, Beijing, China

${ }^{g}$ Center for High Energy Physics, Peking University, Beijing 100871, China

E-mail: mczakon@physik.rwth-aachen. de, aferroglia@citytech.cuny.edu,

dheymes ahep.phy.cam.ac.uk, adm74 acam.ac.uk,

ben.pecjak@durham.ac.uk, d.j.scott@durham.ac.uk,

isasuke.wx@gmail.com, lyangl@gmail.com

In this talk we apply two factorisation theorems to top quark pair production at hadron colliders. The factorisation theorems allow for the resummation of threshold logarithms as well as the resummation of small-mass logarithms in the threshold limit. We combine these two resummed results performing a joint resummation and match to NNLO fixed order results yielding predictions for the pair invariant mass (PIM) and top quark $p_{T}$ distributions at NNLO+NNLL' accuracy. In particular we find the resummed results for the PIM distributions are much more stable with respect to the parametric choice of factorisation scale.

25th International Workshop on Deep Inelastic Scattering and Related Topics

3-7 April 2017

University of Birmingham, Birmingham, UK

* Speaker. 


\section{Introduction}

The top quark, being the heaviest known fundamental particle, is particularly interesting to study. For a start, it couples most strongly with the Higgs boson and as such is tightly connected with the electroweak symmetry breaking of the SM. The larger energy scales now being probed at the LHC may be able to shed new light on this sector or indeed spot deviations from SM predictions. In particular, the study of so-called "boosted" top quarks ${ }^{1}$ may also be a fruitful avenue for exploration with regards to hunting new physics effects. For example, for the pair invariant mass (PIM) distributions CMS [1] has released measurements up to $M=2 \mathrm{TeV}$ and ATLAS [2] up to $M=3 \mathrm{TeV}$, where $M$ is the top pair invariant mass. As such, despite contributing negligibly to the total cross section these high energy tails are no longer merely a corner of phase space, but a region for interesting phenomenological study given the large number of top quark pairs expected over the lifetime of the LHC.

With this in mind, the need for accurate predictions from theory is important. Indeed, many steps have already been taken. In particular, the NNLO predictions for differential distributions were recently calculated in $[3,4,5]$. However the appearance of large logarithmic corrections can plague a fixed order perturbative expansion. For example, in the boosted regime mentioned earlier, logarithms of the ratio of the top mass to the PIM may not constitute small corrections to the cross section. As such, these corrections should be resummed to all orders to yield a converging perturbative expansion. Another potentially dangerous contribution to the cross section comes in the form of threshold logarithms. Such logarithms are dynamically generated by the emission of additional gluons into the final state and become increasingly divergent as the gluon energy tends to zero.

In this talk we present predictions obtained from the combination of two resummed predictions matched to NNLO fixed order results. Specifically, we present the formalism used for the two resummed results as well as the procedure for combining the results and matching to NNLO. Predictions for phenomenology are also presented for the PIM and top quark $p_{T}$ distributions. For the PIM distribution we again assess the difference of the resulting predictions from two different choices of factorisation scale. Results presented here explored in more detail in an upcoming publication [6].

\section{Formalism for Resummation from SCET}

We begin this section by analysing the form of fixed order calculations and highlighting some features mentioned in the introduction. The well known QCD factorisation theorem allows us to write the differential cross section for the production of top quark pairs at the LHC as

$$
\frac{d^{2} \sigma}{d M d \cos \theta}=\frac{8 \pi \beta_{t}}{3 s M} \sum_{i j} \int_{\tau}^{1} \frac{d z}{z} \mathscr{L}_{i j}\left(\tau / z, \mu_{f}\right) C_{i j}\left(z, M, m_{t}, \cos \theta, \mu_{f}\right) .
$$

Here we have presented the differential cross section with respect to the top-pair invariant mass $M$ and the scattering angle $\theta . s$ and $\hat{s}$ denote the hadronic and partonic centre of mass energies

\footnotetext{
${ }^{1}$ Boosted, in this context, refers to the region of phase space where the top pair has a greater invariant mass than the top quark mass itself.
} 
squared respectively. The sum is over initial state partons $i$ and $j$. We have also introduced the variables $\beta_{t}=\sqrt{1-4 m_{t}^{2} / M^{2}}, \tau=M^{2} / s$, and $z=M^{2} / \hat{s}$. The hard-scattering kernels $C_{i j}$ are related to the partonic cross section and can be calculated using standard perturbative techniques while $\mathscr{L}_{i j}$ denotes the parton luminosity and is itself a convolution of the parton distribution functions (PDFs) of the two incoming hadrons.

In calculating perturbative corrections from QCD to the hard-scattering kernels one typically encounters logarithms of the ratios of the different scales characterising the process. The first contributions we consider are plus distributions of the form

$$
\alpha_{s}^{n}\left[\frac{\ln ^{p}(1-z)}{1-z}\right]_{+}, \quad 0 \leq p \leq 2 n-1,
$$

where $n$ denotes the $n$-th order correction to the cross section (the born process begining at $\alpha_{s}^{2}$ ). These distributions become divergent as $z \rightarrow 1$, the limit in which almost all of the incoming partonic energy is in the final state top pair and the phase space for additional gluon emission is highly restricted. As such, we associate these large logarithms with the emission of soft gluons. In addition to regular threshold logarithms, we also wish to consider logarithms of the form $\ln ^{n}\left(m_{t}^{2} / M^{2}\right)$. One might expect such logarithms to become increasingly important as the top-pair invariant mass becomes much larger than the top quark mass itself, i.e. the regime in which the top quarks become highly boosted. We make use of two factorisation theorems which allow the simultaneous resummation of these contributions to all orders in perturbation theory [7, 8]. Both are derived within the Soft Collinear Effective Theory (SCET) framework. The factorisation theorems allow a separation of the scales involved into separate component functions.

The first of these factorisation theorems [7] is derived in the threshold limit $z \rightarrow 1$. The hard scattering kernel factorises as

$$
C_{i j}^{\text {Soft }}=\operatorname{Tr}\left[\mathbf{H}_{i j}^{m}\left(M, m_{t}, \mu_{f}, . .\right) \mathbf{S}_{i j}^{m}\left(\sqrt{\hat{s}}(1-z), m_{t}, \mu_{f}, \ldots\right)\right]+\mathscr{O}(1-z) .
$$

Here $\mathbf{H}_{i j}^{m}$ and $\mathbf{S}_{i j}^{m}$ are the hard and soft functions respectively with the indices $i j$ labelling the partonic channels. The hard and soft functions are matrices in the space of possible colour structures. We see clearly the separation of scales, the hard function with dependence on $M$, while the soft function depends on $\sqrt{\hat{s}}(1-z)$, and as such contains contributions singular as $z \rightarrow 1$. It is worth pointing out that in this soft limit, it is also possible to obtain the transverse momentum $\left(p_{T}\right)$ distribution of the top quark using a simple change of variables. This is possible because the top quarks will be produced back-to-back in the centre of mass frame as soft emissions will not alter their kinematics.

The second factorisation theorem, relevant when $M \gg m_{t}$, is given by [8]

$$
\begin{aligned}
C_{i j}^{\text {Boosted }}= & C_{D}^{2}\left(m_{t}, \mu_{f}\right) \operatorname{Tr}\left[\mathbf{H}_{i j}\left(M, \mu_{f}, . .\right) \mathbf{S}_{i j}\left(\sqrt{\hat{s}}(1-z), \mu_{f}, \ldots\right)\right] \otimes \mathbf{s}_{D}\left(m_{t}(1-z), \mu_{f}\right) \\
& \otimes \mathbf{s}_{D}\left(m_{t}(1-z), \mu_{f}\right) \otimes c_{i j}^{t}\left(z, m_{t}, \mu_{f}\right)+\mathscr{O}(1-z)+\mathscr{O}\left(m_{t} / M\right)
\end{aligned}
$$

where the symbol $\otimes$ denotes a convolution. This factorisation theorem builds on that in Eq. (2.3) and as such is valid to leading power in the limits $z \rightarrow 1$ and $m_{t} / M \rightarrow 0$. Here, the hard and soft functions are now independent of the top mass $m_{t}$ and we have introduced new functions $C_{D}$ and 
$\mathbf{s}_{D}$ which are related to the perturbative heavy-quark fragmentation function. The heavy flavour matching coefficients $c_{i j}^{t}$ arise from the presence of heavy quark loops and in general introduces additional dependence on $\ln \left(m_{t}\right)$. We add these contributions in to the resummed result in fixed order only. Eq. (2.4) thus separate the two hard scales $M$ and $m_{t}$ from each other, and from the soft scales $\sqrt{\hat{s}}(1-z)$ and $m_{t}(1-z)$. Eqs. (2.3) and (2.4) give the starting point for obtaining resummed results. In the original derivation of these, the resummed result was obtained directly in momentum space. However in this instance we choose to work in Mellin space. In Mellin space, convolutions of functions become simple products. The Mellin transformation and its inverse are given by

$$
\tilde{f}(N)=\int_{0}^{1} d x x^{N-1} f(x), \quad f(x)=\frac{1}{2 \pi i} \int_{c-i \infty}^{c+i \infty} d N x^{-N} f(\tilde{N}) .
$$

In the inverse transform $c$ is chosen such that it lies to the right of all singularities in the integrand. In Mellin space, the $z \rightarrow 1$ limit corresponds to $N \rightarrow \infty$ and our scale separation from the two factorisation formulas is given as

$$
\begin{array}{cl}
\text { Mellin-space soft limit: } & \hat{s}, m_{t}^{2} \gg \frac{\hat{s}}{N^{2}}, \\
\text { Mellin-space boosted soft limit: } & \hat{s} \gg m_{t}^{2} \gg \frac{\hat{s}}{N^{2}} \gg \frac{m_{t}^{2}}{N^{2}} .
\end{array}
$$

Deriving and solving the RG equations for each of the matching functions in Eqs. (2.3) and (2.4) we arrive at the resummed form for the hard scattering kernels. In Mellin space, we write these as

$$
\begin{aligned}
\widetilde{c}_{i j}^{\text {Soft }}\left(N, \mu_{f}\right)=\operatorname{Tr}\left[\widetilde{\mathbf{U}}_{i j}^{m}\left(\bar{N}, \mu_{f}, \mu_{h}, \mu_{s}\right) \mathbf{H}_{i j}^{m}\left(\mu_{h}\right) \widetilde{\mathbf{U}}_{i j}^{m \dagger}\left(\bar{N}, \mu_{f}, \mu_{h}, \mu_{s}\right) \widetilde{\mathbf{s}}_{i j}^{m}\left(\ln \frac{M^{2}}{\bar{N}^{2} \mu_{s}^{2}}, \mu_{s}\right)\right] & +\mathscr{O}\left(\frac{1}{N}\right)
\end{aligned}
$$

and

$$
\begin{aligned}
\widetilde{c}_{i j}^{\text {Boosted }}\left(N, \mu_{f}\right) & =\operatorname{Tr}\left[\widetilde{\mathbf{U}}_{i j}\left(\bar{N}, \mu_{f}, \mu_{h}, \mu_{s}\right) \mathbf{H}_{i j}\left(\mu_{h}\right) \widetilde{\mathbf{U}}_{i j}^{\dagger}\left(\bar{N}, \mu_{f}, \mu_{h}, \mu_{s}\right) \widetilde{\mathbf{s}}_{i j}\left(\ln \frac{M^{2}}{\bar{N}^{2} \mu_{s}^{2}}, \mu_{s}\right)\right] \\
\times & \widetilde{U}_{D}^{2}\left(\bar{N}, \mu_{f}, \mu_{d h}, \mu_{d s}\right) C_{D}^{2}\left(m_{t}, \mu_{d h}\right) \widetilde{s}_{D}^{2}\left(\ln \frac{m_{t}}{\bar{N} \mu_{d s}}, \mu_{d s}\right)+\mathscr{O}\left(\frac{1}{N}\right)+\mathscr{O}\left(\frac{m_{t}}{M}\right) .
\end{aligned}
$$

for the threshold resummed and small mass plus threshold resummed results respectively. Here we have removed all dependence of the matching and evolution functions on $M, m_{t}$ and $\cos \theta$. The evolution functions $\mathbf{U}^{m}, \mathbf{U}$ and $U_{D}$ arise from the solutions of the RG equations for each of the matching functions. The massive hard and soft functions can be found to one loop in [7] meaning we can perform threshold resummation to NNLL accuracy. The massless hard and soft functions are known to two loops $[9,10]$ however. In addition, the functions $C_{D}$ and $s_{D}$ were also extracted to NNLO in [8]. Including these two loop matching functions in the boosted soft resummed result yields a resummation accuracy of NNLL' which incorporates a single extra logarithm at each order in perturbation theory compared to NNLL. 
The matching functions $\mathbf{H}^{(m)}, \mathbf{S}^{(m)}, C_{D}$ and $s_{D}$ can now each be evaluated at their own scales $\left(\mu_{h}, \mu_{s}, \mu_{d h}\right.$ and $\mu_{d s}$ respectively) where they are free of large logarithmic corrections. The difference is accounted for via the evolution functions which resum logarithmic contributions involved when running each function to a common scale $\mu_{f}$. In order to free each matching function of large logarithms, we adopt $\mu_{h}=M, \mu_{s}=M / \bar{N}, \mu_{d h}=m_{t}$ and $\mu_{d s}=m_{t} / \bar{N}$ as our canonical default scale choices ${ }^{2}$. The factorization scale $\mu_{f}$ will be correlated with the distribution under consideration and will be specified in each case.

In order to obtain results in momentum space it is necessary to perform the inverse Mellin transform in Eq. (2.5). However, because the soft scales $\mu_{s}$ and $\mu_{d s}$ are chosen directly in Mellin space, the inverse transform encounters a pole for large $N$ related to the running of $\alpha_{s}$. We employ the minimal prescription [11] in order to deal with this. This amounts to choosing the intersection of the contour with the real axis ( $c$ in Eq. (2.5)) to lie to the right of all singularities in the integrand except that arising from the Landau pole at large $N$.

\section{Matching with NNLO Calculations}

So far we have presented two resummed formulas, Eqs (2.8) and (2.9) valid in the soft and boosted soft limits respectively. We would like to combine the predictions obtained from these resummed calculations and additionally match the results to fixed order at NNLO. In this way, we can utilise the full power of the resummation formulas as well as account for corrections subleading in the threshold limit to NNLO. However, we must ensure we do not double count contributions which are common to the fixed order results and the resummed ones. Combining all the results together yields an accuracy denoted NNLO+NNLL' and is achieved through

$$
\begin{aligned}
d \sigma^{\mathrm{NNLO}+\mathrm{NNLL}^{\prime}}=d \sigma^{\mathrm{NNLL}_{b}^{\prime}} & +\left(d \sigma^{\mathrm{NNLL}_{m}}-\left.d \sigma^{\mathrm{NNLL}_{b}}\right|_{\substack{\mu_{d s}=\mu_{s} \\
\mu_{d h}=\mu_{h}}}\right) \\
& +\left(d \sigma^{\mathrm{NNLO}}-\left.d \sigma^{\mathrm{NNLL}_{\mathrm{b}+\mathrm{m}}^{\prime}}\right|_{\substack{\text { Expansion } \\
\text { NNLO }}}\right) .
\end{aligned}
$$

The notation $d \sigma^{\mathrm{NNLL}_{b}^{\prime}}$ denotes predictions obtained using the hard scattering kernel in the boosted soft limit as in Eq. (2.9) at NNLL' accuracy and similarly $d \sigma^{\mathrm{NNLL}_{m}}$ denotes predictions in the threshold limit using Eq. (2.8) to NNLL accuracy. The first line of Eq. (3.1) combines the results from the two resummed predictions. Specifically, the term in brackets on the first line accounts for contributions subleading in the limit $m_{t} / M \rightarrow 0$ which are missed in the boosted soft resummed result but appear in the threshold resummed result. Setting the scales in the matching functions $C_{D}$ and $s_{D}$ equal to the $\mu_{h}$ and $\mu_{s}$ respectively has the effect of undoing the scale separation between the two hard scales $M$ and $m_{t}$ and soft scales $\sqrt{\hat{s}}(1-z)$ and $m_{t}(1-z)$ returning simply the threshold resummed result in the massless limit. This is exactly the contribution double counted when adding on the threshold resummed result and is the origin of the subtraction in the first line. The second line matches the resummed result to NNLO and is simply given by expanding the first line to NNLO and subtracting these contributions out from the exact NNLO contribution, again to avoid double counting such contributions. The NNLO expansion of the joint resummation on the top line

\footnotetext{
${ }^{2}$ Here the notation $\bar{N}=N e^{\gamma_{E}}$ is used to tidy notation.
} 
is a little subtle and an expanded discussion will be available in [6]. However it is worth pointing out that since the matching functions in the threshold resummed result are only known to NLO, the expansion of $d \sigma^{\mathrm{NNLL}_{m}}$ to NNLO leaves dependence on the matching scales $\mu_{h}$ and $\mu_{s}$ in fixed order. The same is true for $d \sigma^{\mathrm{NNLL}_{b}}$ (but not $d \sigma^{\mathrm{NNLL}_{b}^{\prime}}$ where the matching functions are included up to NNLO accuracy).

\section{Numerical Results}
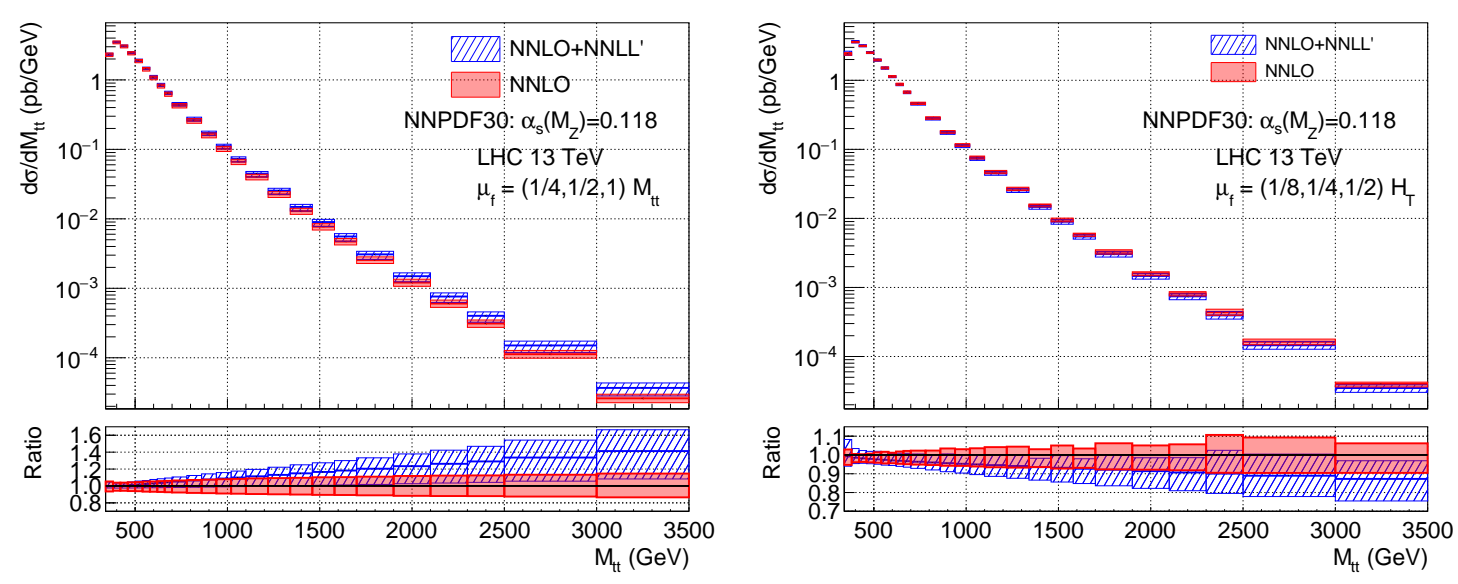

Figure 1: Pair invariant mass distributions obtained using $\mu_{f}=M / 2$ (left) and $\mu_{f}=H_{T} / 4$ (right). Each plot shows predictions from NNLO (red) and NNLO+NNLL' (blue hatched). The lower panel in each plot displays the ratio of the results, normalised to the NNLO prediction.

We now present results obtained from the matching formula in Eq. (3.1). Throughout we use the NNPDF3.0 PDF sets with $\alpha_{s}\left(M_{Z}\right)=0.118$ [12] and set $m_{t}=173.3 \mathrm{GeV}$. Uncertainties are calculated varying each of the scales $\left(\mu_{f}, \mu_{h}, \mu_{s}, \mu_{d h}\right.$ and $\left.\mu_{d s}\right)$ independently by a factor of two up and down, keeping the others fixed and comining the results in quadrature. For the NNLO results we keep $\mu_{f}=\mu_{R}$ at all times. Figure 1 displays predictions for the PIM distributions using two different choices of factorisation scale. In each case we compare the fixed order NNLO and resummed NNLO+NNLL' predictions. The plot on the left shows results obtained using $\mu_{f}=M / 2$. We notice the effect of the resummed result (blue) is to enhance the cross section compared to the fixed order result, particularly in the higher energy bins. In [5] however, it was found that $\mu_{f}=H_{T} / 4$ was a particularly appropriate scale choice in fixed order. We show predictions using this choice of scale on the right hand plot. Here, the resummed result is softer in the tail than its fixed order counterpart. In order to better compare these results we display in Figure 2 the ratios of the fixed order results at the two different scale choices (left) and the corresponding ratios for the resummed results (right). In both cases results are normalised to those with the scale choice $\mu_{f}=H_{T} / 4$. We see that while the fixed order predictions displays a strong dependence on the choice of factorisation scale, the resummed results are generally more stable under such changes.

In Figure 3 we display predictions for the top quark $p_{T}$ distribution using the scale choice $\mu_{f}=m_{T} / 2$. Here we see the resummation has only a limited impact on the resulting $p_{T}$ distribu- 

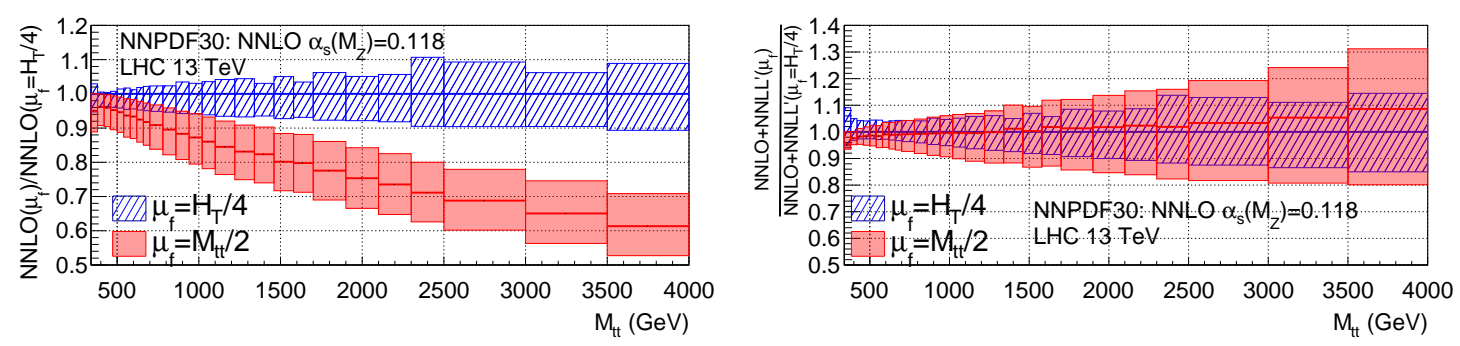

Figure 2: Predictions for the PIM distribution from NNLO fixed order (left) and NNLO+NNLL' (right) with the scale choices $\mu_{f}=H_{T} / 4$ (blue hatched) and $\mu_{f}=M / 2$ (red). Results are normalised to the prediction obtained from using $\mu_{f}=H_{T} / 4$.

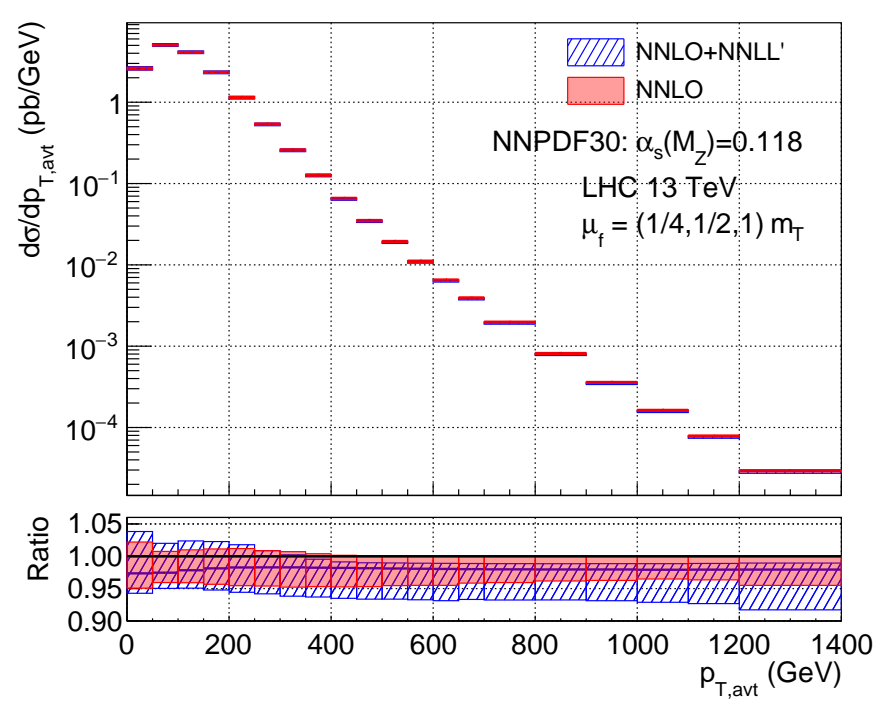

Figure 3: Predictions for the top quark $p_{T}$ distribution at NNLO (red) and NNLO+NNLL' (blue hatched) accuracy. In the lower panel the ratio between the two predictions is taken, normalised to the NNLO result. Predictions are obtained with $\mu_{f}=m_{T} / 2$.

tion. There is a slight softening of the central value, however both predictions lie well within the uncertainty band of each other.

As the experimental uncertainties in the high energy tails of distributions become smaller, the need for ever more accurate theory predictions will grow. We have taken some steps in this direction here, supplementing the fixed order NNLO calculations with resummed results.

\section{References}

[1] CMS Collaboration [CMS Collaboration], CMS-PAS-TOP-16-008.

[2] The ATLAS collaboration [ATLAS Collaboration], ATLAS-CONF-2016-100.

[3] M. Czakon, D. Heymes and A. Mitov, Phys. Rev. Lett. 116, no. 8, 082003 (2016) doi:10.1103/PhysRevLett.116.082003 [arXiv:1511.00549 [hep-ph]]. 
[4] M. Czakon, P. Fiedler, D. Heymes and A. Mitov, JHEP 1605, 034 (2016) doi:10.1007/JHEP05(2016)034 [arXiv:1601.05375 [hep-ph]].

[5] M. Czakon, D. Heymes and A. Mitov, JHEP 1704, 071 (2017) doi:10.1007/JHEP04(2017)071 [arXiv:1606.03350 [hep-ph]].

[6] M. Czakon, A. Ferroglia, D. Heymes, A. Mitov, B. Pecjak, D. Scott, X. Wang and L. Yang, In preparation

[7] V. Ahrens, A. Ferroglia, M. Neubert, B. D. Pecjak and L. L. Yang, JHEP 1009, 097 (2010) doi:10.1007/JHEP09(2010)097 [arXiv:1003.5827 [hep-ph]].

[8] A. Ferroglia, B. D. Pecjak and L. L. Yang, Phys. Rev. D 86, 034010 (2012) doi:10.1103/PhysRevD.86.034010 [arXiv:1205.3662 [hep-ph]].

[9] A. Broggio, A. Ferroglia, B. D. Pecjak and Z. Zhang, JHEP 1412, 005 (2014) doi:10.1007/JHEP12(2014)005 [arXiv:1409.5294 [hep-ph]].

[10] A. Ferroglia, B. D. Pecjak, L. L. Yang, B. D. Pecjak and L. L. Yang, JHEP 1210, 180 (2012) doi:10.1007/JHEP10(2012)180 [arXiv:1207.4798 [hep-ph]].

[11] S. Catani, M. L. Mangano, P. Nason and L. Trentadue, Nucl. Phys. B 478, 273 (1996) doi:10.1016/0550-3213(96)00399-9 [hep-ph/9604351].

[12] R. D. Ball et al. [NNPDF Collaboration], JHEP 1504, 040 (2015) doi:10.1007/JHEP04(2015)040 [arXiv:1410.8849 [hep-ph]]. 